\title{
Low temperature magnetic behaviour of glass-covered magnetic microwires with gradient nanocrystalline microstructure
}

\author{
I. G. Serrano, A. Hernando, and P. Marín \\ Instituto de Magnetismo Aplicado, UCM-ADIF-CSIC, P.O. Box 155 las Rozas, Madrid 28230, Spain
}

(Received 30 December 2013; accepted 5 January 2014; published online 17 January 2014)

\begin{abstract}
Slow nanocrystallization driving dynamics can be affected by the combination of two factors: sample residual stresses and sample geometry. This effect is evidenced at the initial stages of nanocrystallization of amorphous $\mathrm{CoFeSiBCuNb}$ magnetic microwires. Transmission electron microscopy observations indicate how crystallization at temperatures between 730 and $780 \mathrm{~K}$ results in a graded microstructure where the crystallization at the surface skin of the microwire, which remains almost amorphous, differs from that of the middle, where elongated grains are observed, and inner regions. However, samples annealed at higher temperatures present a homogeneous microstructure. The effect of gradient microstructure on magnetic properties has been also analyzed and a loss of bistable magnetic behaviour at low temperatures, from that obtained in the amorphous and fully nanocrystallized sample, has been observed and ascribed to changes in sign of magnetostriction for measuring temperatures below $100 \mathrm{~K}$. (C) 2014 AIP Publishing LLC.

[http://dx.doi.org/10.1063/1.4862540]
\end{abstract}

High performance materials are those essential to important modern applications as ultra-sensitive sensors, actuators and transducers, space power generators, high density magnetic storage media, electromagnetic waves shielding, etc., that should work in extreme environments. However, the full advantage that these materials can provide is strongly dependent on composition and microstructure. ${ }^{1}$ On the other hand, the concept of gradient materials that refer to the introduction of engineered chemical composition and/or microstructure parameter profiles has been used to tailor materials with desired properties and has gained much attention due to their potential applications. ${ }^{2-5}$ In the case of magnetic materials, high-performance means operating at high frequency, high power densities, and high temperatures in extreme environments. $^{6-8}$ These applications require soft magnetic materials not only preserving excellent conductivity, high permeability and saturation magnetization, low coercivity, low eddy-current losses, and high Curie temperatures ${ }^{6-9}$ but also the necessary mechanical strength (i.e., toughness) for processing.

Nanocrystallization from the amorphous state has become a promising method currently available for producing desirable amorphous-nanocrystalline structures with high magnetic characteristics but, generally, these processes involve mechanical properties deterioration associated to embrittlement and ultimate rupture strength decrease. ${ }^{10}$ Consequently, new graded processes, focused to obtain high-performance nanocrystalline magnetic materials with superior mechanical properties combined with excellent magnetic and/or electronic properties, are going on. ${ }^{6-8}$ These graded mechanisms should be based on the fact that amorphous solids are thermodynamically metastable and amorphous to-crystalline phase transformation will be stimulated if sufficient energy to drive this process is supplied. Thermal annealing is a common method to ahead these transformations. ${ }^{11}$ Mechanical energy is another factor resulting in crystallization progress of amorphous and bulk metallic glasses, such as high pressure, ${ }^{12,13}$ cold-rolling, ${ }^{14}$ tension, ${ }^{15}$ compression and bending. ${ }^{16}$ So, nanocrystallization can be driven by applying stress and temperature, i.e., thermal energy activates the phase transformation and mechanical energy promotes this process.

Remanent stress in as-cast samples may condition the nanocrystallization. Despite the uncertainties connected with the applicability of the classical nucleation theory to crystallization of glass-forming liquids, one can expect that the effect of stresses on nucleation will be, in most cases, much more significant than on growth. Schmelzer et al. ${ }^{17}$ have shown how, in general, elastic stress effects are of importance only in temperature ranges where the growth rate is low and its effect depends significantly on the ratio of the thermodynamic driving force, $\Delta \mu$, and the elastic stress parameter, $\varepsilon_{\mathrm{o}}$. Elastic stresses can affect crystal growth rates only at temperatures below a decoupling temperature. Magnetic energy has been stated as other factor involving changes in nanocrystallization driving dynamics. ${ }^{18}$ It has been shown how nanocrystalline grains can be directionally ordered by annealing of $\mathrm{Fe}_{28.5} \mathrm{Co}_{45} \mathrm{Si}_{13.5} \mathrm{Cu}_{1} \mathrm{Nb}_{3}$ ribbons in the presence of magnetic field. The intensity of the field allows controlling different degrees of order. This happens when the annealing temperature corresponds to the first stages of nanocrystallization process. The field effect can be described by a relative increase of the crystallized volume fraction and a linear alignment of the nucleated grains. The energy barrier for nucleation is directionally affected by the applied field. A combination of Zeeman, magnetostatic, and magnetocrystalline free energy terms were proposed to give rise to the observed directional order. Based on this work, Tang et al. show a graded process based on directional magnetic field annealing of amorphous materials using cobalt-rich alloys ${ }^{19}$ for the fabrication of gradient-structural magnetic materials. This work shows how longitudinal magnetic field presence results in a gradient-like 
microstructure across the ribbon; on the contrary, a transverse field favors the formation of middle-range directionally ordered phases.

It is well known that amorphous materials, obtained by rapid quenching, present residual stresses inherent to fabrication process and sample geometry that have been thoroughly studied in the case of glass covered magnetic microwires due to its great influence on magnetic anisotropy ${ }^{20}$ These microwires, prepared by quenching and drawing technique, consist of a magnetic nucleus with diameter from 1 to $30 \mu \mathrm{m}$ coated by a Pyrex-like glass with thickness of $2-20 \mu \mathrm{m}$. Due to their amorphous nature, magnetoelastic and shape anisotropy contributions govern their magnetic properties. As a result of abovementioned anisotropies, the domain structure of microwires with positive magnetostriction consists of single axial domain and their magnetization process is characteristic of the bistable behavior. Nanocrystalline microwires have been the subject of different studies. ${ }^{21,22}$ It has been observed how in the annealing process towards nanocrystallization the microwires undergo strong stresses due to the different thermal expansion coefficients of the metallic nucleus and the glass coating. In general, the formation of phases with higher pack densities is energetically favoured by stress or pressure. This influence is observed in Finemet microwires, where a non-magnetic $\alpha$-Fe phase is observed instead of the desired $\alpha-\mathrm{Fe}(\mathrm{Si}),{ }^{21}$ Komova et al. show how, contrary to expectations, further increase of annealing temperature leads to vanishing Bragg peaks. At high temperature, very high stresses are induced in the metallic nucleus resulting in the suppression of atomic mobility. The aim of the present work is to evaluate the counterbalance between thermal and mechanical energy influence on nanocrystallization process. The magnetic behavior of the samples has been also analyzed.

Glass-covered amorphous microwires of nominal composition $\mathrm{Fe}_{28.5} \mathrm{Co}_{45} \mathrm{Si}_{13.5} \mathrm{~B}_{9} \mathrm{Cu}_{1} \mathrm{Nb}_{3}$ and metal core diameter of $10 \mu \mathrm{m}$ and $8 \mu \mathrm{m}$ thick glass cover were obtained by Taylor's technique. $^{20}$ This paper refers to as-cast sample (sample A) and isochronously $(1 \mathrm{~h}$ ) annealed, at $693 \mathrm{~K}$ (sample B), $733 \mathrm{~K}$ (sample C), $773 \mathrm{~K}$ (sample D), and $793 \mathrm{~K}$ (sample E) in argon atmosphere. X-ray diffractograms have been obtained by using copper $\lambda=0.1541 \mathrm{~nm}$ (Siemens D5000). Microstructural study has been performed by means of Transmission Electron Microscopy (JEOL JEM 3000FX) and electron energy loss

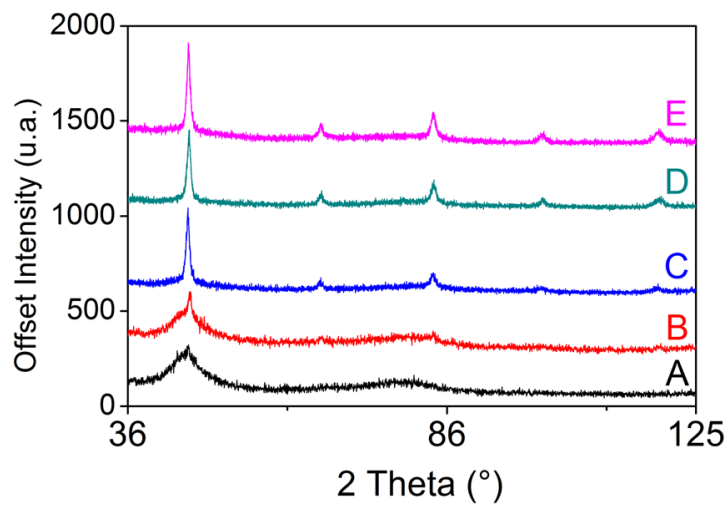

FIG. 1. X-ray diffractograms for FeCoSiBCuNb microwires as-cast (A), annealed at: $693 \mathrm{~K}(\mathrm{~B}), 733 \mathrm{~K}(\mathrm{C}), 773 \mathrm{~K}$ (D), and $793 \mathrm{~K}(\mathrm{E})$.

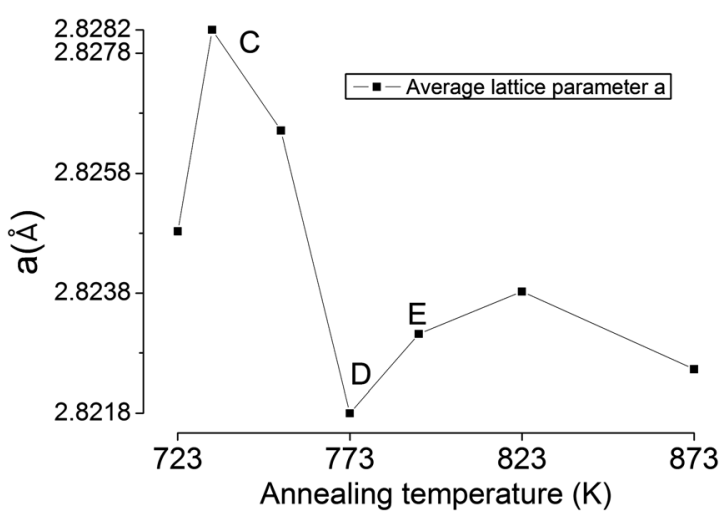

FIG. 2. Calculated average lattice parameter $a$ as a function of annealing temperature. Labels represent the samples annealed at $733 \mathrm{~K}$ (C), $773 \mathrm{~K}$ (D), and $793 \mathrm{~K}(\mathrm{E})$.

spectroscopy (EELS). Microwire slices of $60 \mathrm{~nm}$ thicknesses were obtained using Ultramicrotome (Reichert Jung Ultracut E). Low temperature hysteresis loops have been obtained by means of VSM-PPMS 6000 Quantum Design magnetometer.

The annealing influence on X-ray diffractograms is shown in Figure 1 and analyzed as follows. Samples A and $\mathrm{B}$ microstructures are predominantly amorphous although a slight crystallization trace positioned in $2 \theta=45.69^{\circ}$ is observed for B. Annealing temperature increase results in an iron based nanocrystalline microstructure with a mainpeak positioned in $2 \theta=45.41^{\circ}$. In the particular case of sample $\mathrm{C}$, the shown traces, by means of Scherrer formula, have been associated to $\mathrm{Fe}_{3} \mathrm{Si}$ grains with an estimated size of $13.7 \mathrm{~nm}$. A peak shifting to $45.5^{\circ}$ and increase in high is observed for samples D and E.

Figure 2 shows the variation of the mean lattice constant, $a$, obtained from the X-ray diffractograms, as a function of annealing temperature. It was calculated considering as a bcc lattice. The reflection peaks can be associated to bcc

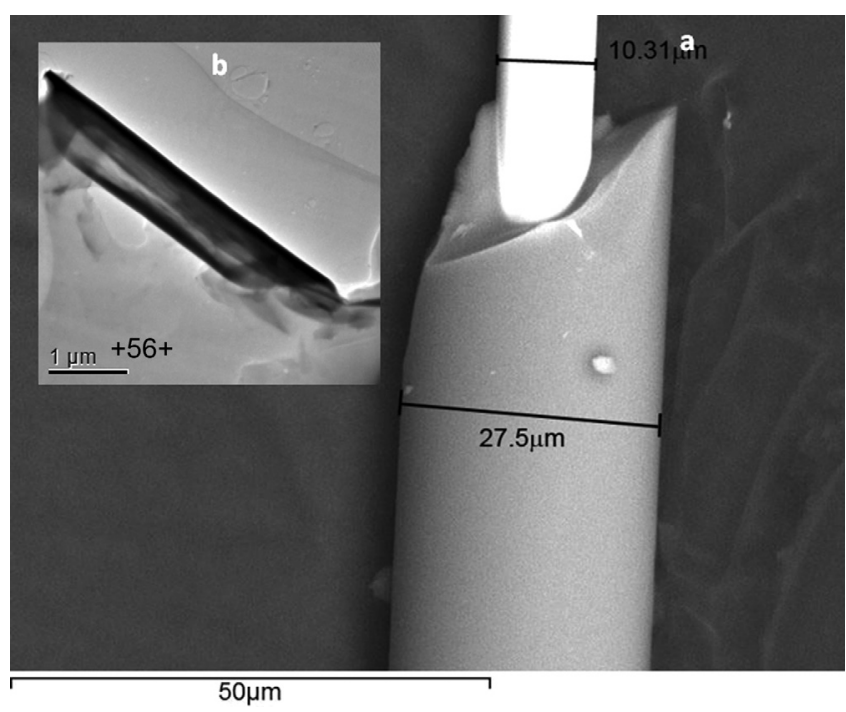

FIG. 3. A SEM microphotography for as-cast FeCoSiBCuNb microwire showing the dimensions of core and Pyrex coating (a), and a TEM microphotography showing a microwire transversal cut preparation for TEM (b), note that the microwire is curling due to the cut. 

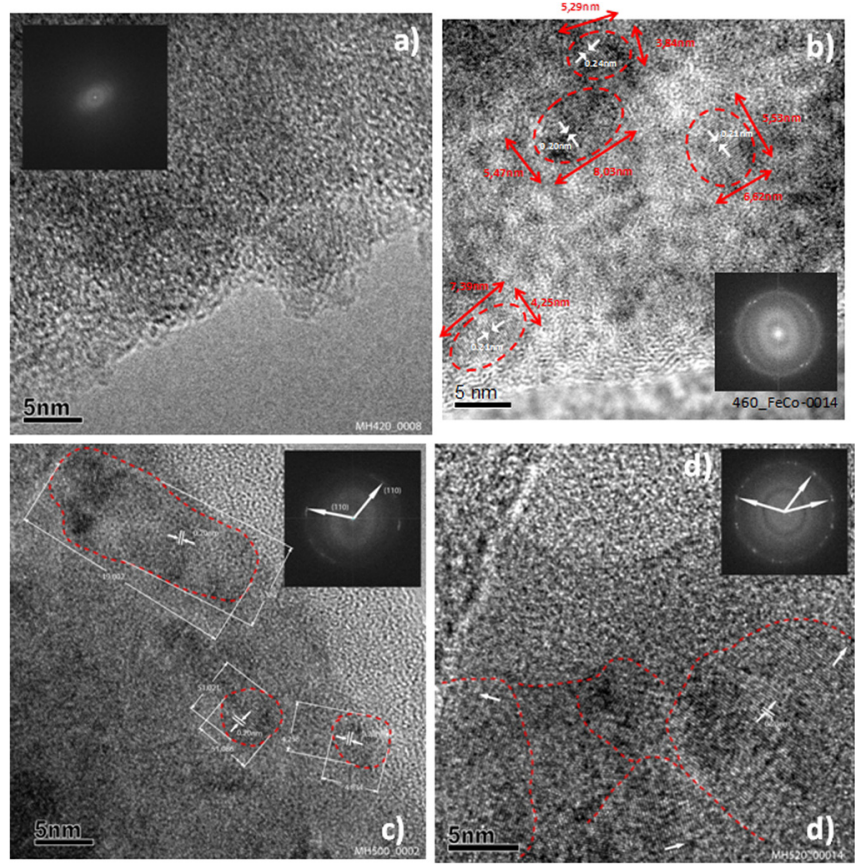

FIG. 4. A transversal cut TEM microphotographs of the $\mathrm{FeCoSiBCuNb}$ microwires showing the evolution of crystallization percentage for $1 \mathrm{~h}$ annealing at $693 \mathrm{~K}$ (a), $733 \mathrm{~K}$ (b), $773 \mathrm{~K}$ (c), and $793 \mathrm{~K}$ (d). The inset of each one shows the FFT.

Fe rich phase or Co rich phase. ${ }^{11}$ A significant drop for lattice parameter, a, is observed for sample D. Lower values of $a$ suggest higher amount of cobalt in the phases. ${ }^{23,24}$

Figure 3 shows a SEM microphotography of a $\mathrm{Fe}_{28.5} \mathrm{Co}_{45}$ $\mathrm{Si}_{13.5} \mathrm{~B}_{9} \mathrm{Cu}_{1} \mathrm{Nb}_{3}$ microwire, the inset shows transversal cut of the microwire.

Electron micrographs for samples B, C, D, and E, respectively, are shown in Figure 4. The amorphous character of sample B (Figure 4(a)) is confirmed by the fast Fourier Transform (FFT) plot. An initial stage of crystallization, with low crystalline fraction as stated by FFT, is found in the sample annealed at $733 \mathrm{~K}$ (sample C) (Figure 4(b)). The observed nanograins are elongated, with an average size of $6 \times 4 \mathrm{~nm}$, and almost randomly distributed and embedded in an amorphous matrix, with certain tendency to parallel orientation. For the sample annealed at $773 \mathrm{~K}$ (sample D) (Figure 4(c)), a greater proportion of crystalline material has been found. These grains, higher than that observed in the previous case, are elongated with an average size of $12 \times 8 \mathrm{~nm}$. This grain elongation tends to be parallel to the edge of the sample. Further annealing at $793 \mathrm{~K}$ (sample E) (Figure 4(d)) results in a totally crystalline sample where the differences between crystalline planes orientation offer the possibility to distinguish one zone to other.

Figure 5(a) shows an enhanced image of a particle observed in sample $\mathrm{C}$ and its corresponding FT. The lattice distance, $0.20 \mathrm{~nm}$, and the (110) direction is arrowed. Figure 5 (b) is a less magnified image of $793 \mathrm{~K}$ (sample E) with the aim to show the amorphous region between sample border and crystalline area. The arrow indicates an intermediate amorphous region between sample border and crystalline zone.

The wide range of observed microstructures is evident when analyzing the influence of temperature on samples magnetic behavior. Hysteresis loops of positive magnetostrictive amorphous microwires used to be bistable presenting a single Barkhausen jump between two stable remanent states. ${ }^{25}$ In the amorphous state, a quite simple domain structure, determined by magnetostrictive and shape energy terms, is responsible for this behavior. It consists of a singledomain ${ }^{26}$ inner core with magnetization oriented parallel to the wire axis and surrounded by an outer domain shell with the magnetization oriented perpendicular to the wire axis with closure domains at the end of the microwire. This configuration results in the specific magnetization process when the wire is magnetized in a field parallel to its axis. Then, at the switching field, the magnetization reversal process runs in a single giant Barkhausen jump as a domain wall depins from the closure structure at one of the ends and propagates along the entire microwire. ${ }^{27}$ The depinning of a domain wall from the closure structure at one end is responsible for the coercivity mechanism. The temperature dependence of the switching field can be interpreted in terms of two pinning mechanisms of closure domain wall. ${ }^{25,28,29}$ First, there is a magnetoelastic pinning of the closure domain structure coming from the mechanical stresses present in the microwire; second, pinning of the closure domain at the atomic level defects responsible for the increase of the switching field at low temperatures.

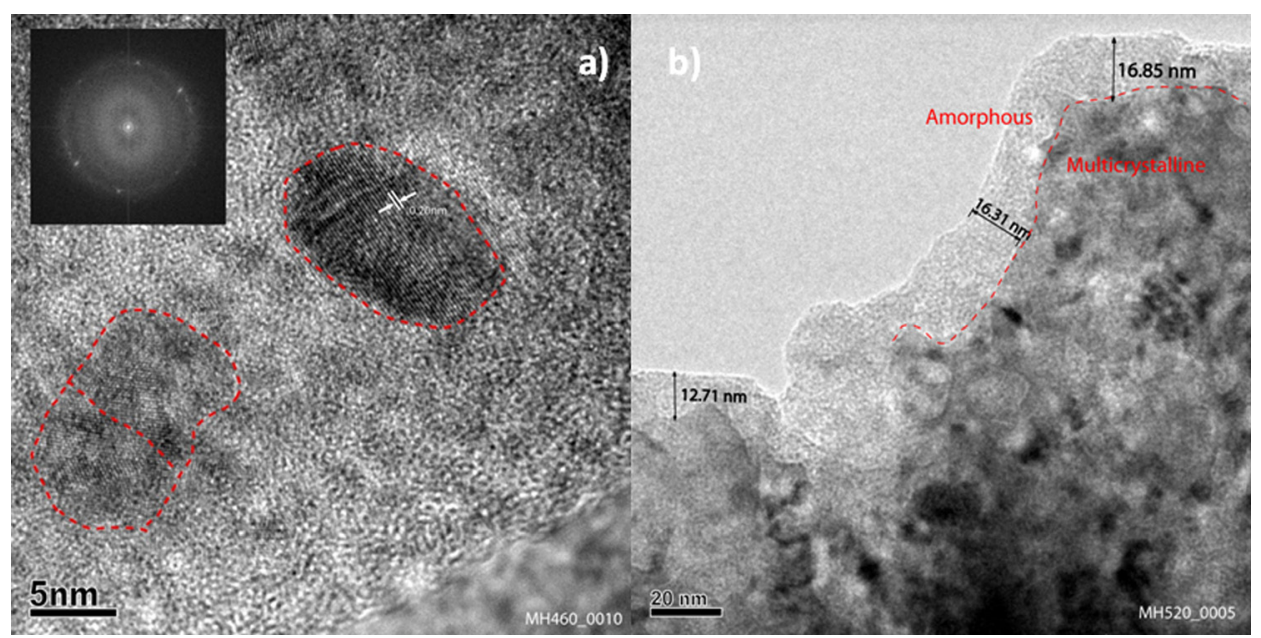

FIG. 5. Enhanced image of a particle observed in sample D and its corresponding Fourier transformation (a). TEM microphotography for FeCoSiBCuNb microwire for $1 \mathrm{~h}$ annealed at $793 \mathrm{~K}$ showing a detail of the amorphous border in contrast with a core almost crystallized (b). 


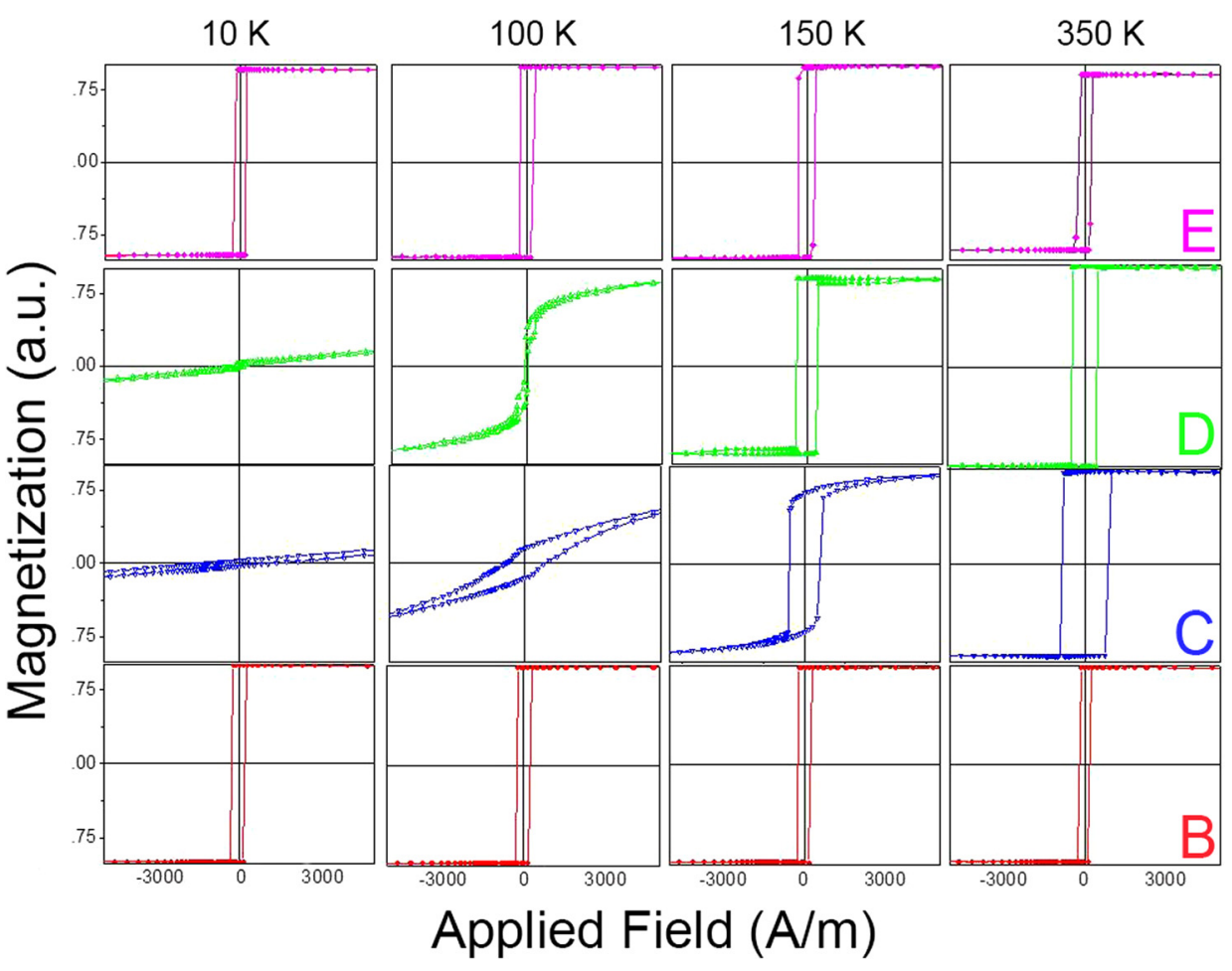

FIG. 6. Hysteresis loops evolution with measuring temperature for $\mathrm{FeCOSiBCuNb}$ microwire samples annealed at $693 \mathrm{~K}$ (B), $733 \mathrm{~K}(\mathrm{C}), 773 \mathrm{~K}(\mathrm{D})$, and $793 \mathrm{~K}$ (E).
The present work analyzes the effects of measuring temperature on the bistability for each and every one of the obtained microstructures. An overview of hysteresis loops, for annealed samples, is shown in Figure 6. All samples measured at room temperature exhibit bistable behavior. It should be noted that there is a clear increase in the critical switching field for those partially crystallized, this effect being more significant for those treated at $733 \mathrm{~K}$ (sample C). As shown, crystallization process starts close to this temperature. However, crystalline precipitates are small, having a long distance between them. Hence, they act the role of the pinning centers for the domain wall and it results in the increase of RT switching field annealing at $793 \mathrm{~K}$, which leads to the appearance of homogeneous nanocrystalline structure. Exchange interaction of crystalline grains leads to the averaging out of magnetocrystalline anisotropy that results in a magnetic softness (switching field decreases). ${ }^{30}$

As observed by other authors, ${ }^{27}$ the homogeneous samples, i.e., amorphous (637 K annealed (sample B)) and annealed at $793 \mathrm{~K}$ (sample E), remain bistable at low temperature. The slight increase of switching field at low temperature should be ascribed to magnetoelastic pinning increase through magnetostriction constant. Partially crystallized samples present the more surprising behavior (samples C and D) (Figure 6(c)). In this case, temperature drop involves bistability loss and a temperature around $100 \mathrm{~K}$ can be considered as a transition temperature $\left(T_{t}\right)$ between two well differentiated magnetic states. The longitudinal anisotropy vanishes as temperature decreases modifying magnetic domain orientation towards a domain structure perpendicular to the axial direction resulting in a microwire longitudinal anisotropy decrease. The effect is more striking for sample annealed at $773 \mathrm{~K}$ (sample C).
Figure 7 shows coercivity evolution with measuring temperature (zoom in the inset). Hollow symbols correspond to non-bistable hysteresis loops. The most important conclusions that can be drawn from this figure are as follows. Samples with homogeneous microstructure (B and E) remain bistable in the wide temperature range showing coercivity decrease with increasing measuring temperature. Inhomogeneous microwires (C and $\mathrm{D})$, both behaving in an equivalent way, present a minimum around the transition temperature, $T_{t}$. The inset shows in detail this minimum for samples $\mathrm{D}$ and $\mathrm{C}$ that run at higher coercivity ranges.

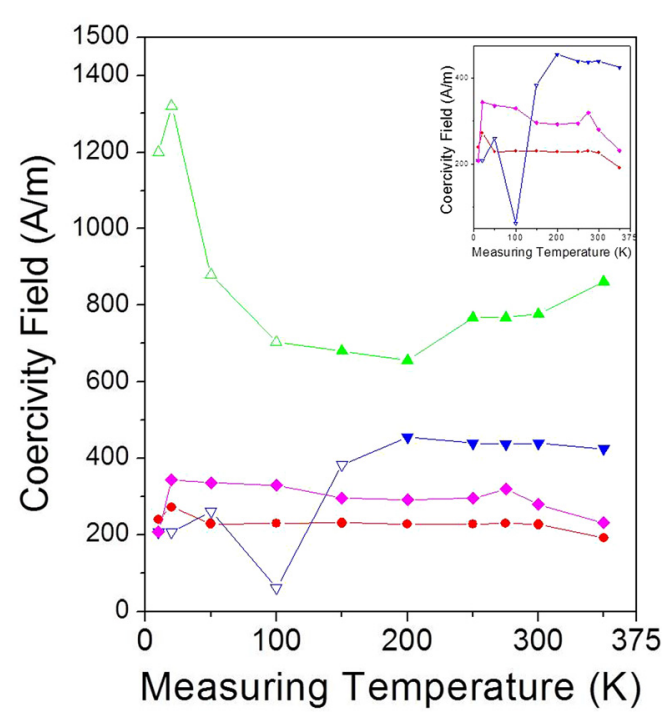

FIG. 7. Influence of measuring temperature on coercivity field for FeCoSiBCuNb annealed under $693 \mathrm{~K}(\bullet$ (red)), $733 \mathrm{~K}(\boldsymbol{\nabla}, \nabla$ (blue)), $773 \mathrm{~K}$ $(\boldsymbol{\Delta}, \triangle($ green $))$, and $793 \mathrm{~K}(\bullet$ (pink)) microwire samples. (Hollow symbols represent non-bistable hysteresis loops.) 
Let us analyze the possible origin of the experimental results reported here. Amorphous solids are thermodynamically metastable and will be crystallized into one or more metastable or stable polycrystalline phases upon thermal annealing or mechanical activation. The crystallization kinetics of amorphous solids induced by thermal annealing is found to be sensitive to composition of the materials as well as the annealing conditions such as temperature and heating rate. As reported by Marin et al., ${ }^{18}$ crystallization kinetics of selected $\mathrm{Fe}_{28.5} \mathrm{Co}_{45} \mathrm{Si}_{13.5} \mathrm{~B}_{9} \mathrm{Cu}_{1} \mathrm{Nb}_{3}$ composition presents a first crystallization process at $724 \mathrm{~K}$ associated with partial crystallization of nanograins with expected low crystallization dynamic and growth rate with a thermodynamic driving force affected by elastic stress parameter.

In the case of glass-coated microwires, we have to consider the following mechanical stresses that could have influence on nanocrystallization process: (i) thermoelastic stresses from the temperature gradient during quenching; (ii) drawing stresses, tensile in nature, originated by the extraction process; and (iii) stresses coming from the different thermal expansion coefficients of metallic nucleus and insulating coating. Average value of stresses in glass-coated microwires is of the order of 400-500 MPa. Different authors ${ }^{20,31-33}$ have calculated the stress distributions. Baranov et al. considered that most stresses actually arise from the differential thermal expansion coefficients, thus obtaining complex axial, $\sigma_{\mathrm{zz}}$, radial, $\sigma_{\mathrm{rr}}$, and azimuthal, $\sigma_{\varphi \varphi}$, stress contributions. The centre of the wire $(r=0)$ constitutes a singularity in which all the stress components must be null in order to ensure the equilibrium in this point. The total internal stress distribution has two zones: the first one in which the axial component, $\sigma_{\mathrm{zz}}$, is the highest and positive (from the centre of the wire to a distance $\left.\mathrm{r}=0.7 \mathrm{r}_{\mathrm{m}}\right)$ and where $\sigma_{\mathrm{rr}}$ and $\sigma_{\varphi \varphi}$ are almost null and the second zone $\left(0.7 \mathrm{r}_{\mathrm{m}}<\mathrm{r}<\mathrm{r}_{\mathrm{m}}\right)$ dominated by the decrease of the axial component towards zero and dominated by the negative value of the circumferential stress component that decreases from zero to GPa units.

In an infinite homogeneous and relaxed amorphous, the grain nucleation should be randomly distributed but as demonstrated in Ref. 18 an external agent as magnetic field can directionally affect the energy barrier for nucleation. This stress distribution should also disturb the thermal stability of amorphous microwires, affecting the energy barriers by decreasing and/or increasing the crystallization temperature for certain directions. ${ }^{34}$ This suggests a coupling effect of the stress on the crystallization during annealing. This coupling effect on the nucleation and growth of the crystals should be related with the elongated shape of the nanocrystals along the azimuthal direction and with the amorphous area between sample border and crystallized area effects observed in Figure 5.

The complex evolution of switching field with measuring temperature of graded microstructures (samples $\mathrm{C}$ and D) should be understood taking into account the counterbalance between the two mechanisms responsible for bistability, i.e., magnetoelastic and atomic level defects pinning of closure domain.

The magnetoelastic pinning is strongly dependent on magnetostriction evolution with temperature. The $\mathrm{FeCoSiBCuNb}$ amorphous composition under study should have positive magnetostriction at room temperature. As shown by x-ray diffraction, a primary crystallization process results in the formation of $\mathrm{Fe}(\mathrm{Co}) \mathrm{Si}$ nanocrystallites embedded in an amorphous matrix. This primary crystallization would alter residual amorphous microstructure resulting in $\mathrm{Co}$ enriched $\mathrm{Co}(\mathrm{Fe}) \mathrm{SiBNb}$. The saturation magnetostriction of this residual amorphous should change in sign with decreasing temperature. ${ }^{35,36}$ At a certain temperature, the consequence would be the closure domain depinning with the consequent bistability loss associated to a change in the magnetization process to domain wall movements.

The obtained lattice parameters shown in Figure 2 are related to cobalt level in the crystalline grains, and consequently to the amorphous matrix cobalt amount. Sample C presents a higher lattice parameter than D. This corresponds to a less amount of cobalt in the crystalline grains, so it is expected that the amount of cobalt in amorphous matrix to be higher for sample $\mathrm{C}$. This would explain why bistability loss is observed from $150 \mathrm{~K}$ in sample $\mathrm{C}$ in contrast with sample D, which lose the bistability around $100 \mathrm{~K}$. In this last case, a higher cobalt amount in the crystalline lattice is expected.

In conclusion, the observed loss of magnetic bistability at low temperatures is associated to a graded nanocrystalline microstructure, obtained by highly controlled thermal treatments with slow crystallization dynamics hardly influenced by residual mechanical stresses, combined with a change on amorphous residual matrix saturation magnetostriction constant.

This work has been supported by the MEC of Spain (Consolider-Ingenio 2010 Contract Nos. CSD2007-0010 and MAT2009-14741-C02-01), (S2009 MAT 1726 and Projects TSI-020100-2011-280, IPT-2011-0893-420000).

${ }^{1}$ U. Täffner, V. Carle, U. Schäfer, and M. J. Hoffmann, Preparation and Microstructural Analysis of High-Performance Ceramics (ASM International, 2004).

${ }^{2}$ C. P. Wang, X. J. Liu, I. Ohnuma, R. Kainuma, and K. Ishida, Science 297, 990 (2002).

${ }^{3}$ T. Ishigure, E. Nihei, and Y. Koike, Appl. Opt. 35, 2048 (1996).

${ }^{4}$ A. Kumar, G. Li, Z. Hong, and Y. Yang, Nanotechnology 20, 165202 (2009).

${ }^{5}$ B. Kieback, A. Neubrand, and H. Riedel, Mater. Sci. Eng., A 362, 81 (2003).

${ }^{6}$ M. E. Mchenry and D. E. Laughlin, Acta Mater. 48, 223 (2000).

${ }^{7}$ J. Long, M. McHenry, D. P. Urciuoli, V. Keylin, J. Huth, and T. E. Salem, J. Appl. Phys. 103, 07 E705 (2008).

${ }^{8}$ K. J. Miller, A. Leary, S. J. Kernion, A. Wise, D. E. Laughlin, M. E. McHenry, V. Keylin, and J. Huth, J. Appl. Phys. 107, 09A316 (2010).

${ }^{9}$ A. Makino, H. Men, T. Kubota, K. Yubuta, and A. Inoue, J. Appl. Phys. 105, 07A308 (2009).

${ }^{10}$ T. M. Heil, K. J. Wahl, A. C. Lewis, J. D. Mattison, and M. A. Willard, Appl. Phys. Lett. 90, 212508 (2007).

${ }^{11}$ G. Herzer, IEEE Trans. Magn. 25, 3327 (1989).

${ }^{12}$ Z. X. Wang, F. Y. Li, M. X. Pan, D. Q. Zhao, and W. H. Wang, J. Alloys Compd. 388, 262 (2005).

${ }^{13}$ Z. Wang, D. Zhao, M. Pan, P. Wen, W. Wang, T. Okada, and W. Utsumi, Phys. Rev. B 69, 092202 (2004).

${ }^{14}$ R. J. Hebert and J. H. Perepezko, Mater. Sci. Eng., A 375-377, 728 (2004). 
${ }^{15}$ S.-W. Lee, M.-Y. Huh, E. Fleury, and J.-C. Lee, Acta Mater. 54, 349 (2006).

${ }^{16}$ H. Chen, Y. He, G. J. Shiflet, and S. J. Poon, Nature 367, 541 (1994).

${ }^{17}$ J. W. P. Schmelzer, E. D. Zanotto, I. Avramov, and V. M. Fokin, J. Non. Cryst. Solids 352, 434 (2006).

${ }^{18}$ P. Marín, M. López, A. Vlad, A. Hernando, M. L. Ruiz-González, and J. M. González-Calbet, Appl. Phys. Lett. 89, 033508 (2006).

${ }^{19}$ Z. Tang, Y. Song, Q. Sun, T. Zhang, and Y. Jiang, Nanoscale 4, 386 (2012).

${ }^{20}$ H. Chiriac and T. A. Óvári, Prog. Mater. Sci. 40, 333 (1996).

${ }^{21}$ E. Komova, M. Varga, R. Varga, P. Vojtanik, J. Bednarcik, J. Kovac, M. Provencio, and M. Vázquez, Appl. Phys. Lett. 93, 062502 (2008).

${ }^{22}$ C. Garcia, A. Zhukov, J. Gonzalez, V. Zhukova, R. Varga, J. J. del Val, V. Larin, A. Chizhik, and J. M. Blanco, J. Appl. Phys. 99, 08F116 (2006).

${ }^{23}$ C. Gómez-Polo, P. Marín, L. Pascual, A. Hernando, and M. Vázquez, Phys. Rev. B 65, 024433 (2001).

${ }^{24}$ V. Niculescu and J. I. Budnick, Phys. Rev. B 19, 452 (1979).

${ }^{25}$ R. Varga, K. Garcia, M. Vázquez, A. Zhukov, and P. Vojtanik, Phys. Rev. B 70, 024402 (2004).
${ }^{26}$ A. P. Zhukov, M. Vfizquez, H. Chiriac, and V. Larin, J. Magn. Magn. Mater. 151, 132 (1995).

${ }^{27}$ C. Gómez-Polo, M. Vázquez, and D.-X. Chen, Appl. Phys. Lett. 62, 108 (1993).

${ }^{28}$ H. Kronmüller, Phys. Status Solidi 127, 531 (1985).

${ }^{29}$ R. Varga, K. Garcia, M. Vázquez, and P. Vojtanik, Phys. Rev. Lett. 94, 017201 (2005).

${ }^{30}$ M. Vázquez, P. Marín, H. A. Davies, and A. O. Olofinjana, Appl. Phys. Lett. 64, 3184 (1994).

${ }^{31}$ M. Vázquez, "Advanced magnetic microwires," in Handbook of Magnetism and Advanced Magnetic Materials, edited by H. Kronmüller and S. Parkin (Wiley, 2007).

${ }^{32}$ S. A. Baranov, V. N. Berzhanski, and S. K. Zotov, Fiz. Met. i Metalloved. 67, 73 (1989).

${ }^{33}$ J. Velázquez, M. Vázquez, and A. P. Zhukov, J. Mater. Res. 11, 2499 (1996).

${ }^{34} \mathrm{~F}$. Ye and K. Lu, Phys. Rev. B 60, 7018 (1999).

${ }^{35}$ A. Hernando, V. Madurga, C. N. de Villavicencio, and M. Vázquez, Appl. Phys. Lett. 45, 802 (1984).

${ }^{36}$ R. C. O'Handley, Phys. Rev. B 18, 930 (1978). 